\title{
Impact of Hypertonic Saline Solutions on Sputum Expectoration and Their Safety Profile in Patients with Bronchiectasis: A Randomized Crossover Trial
}

\author{
Beatriz Herrero-Cortina, PT, MSc, Victoria Alcaraz, PT, MSc, \\ Jordi Vilaró, $\mathrm{PT}, \mathrm{PhD}^{3}$, Antoni Torres, MD, $\mathrm{PhD}^{4}$, and Eva Polverino, $\mathrm{MD}, \mathrm{PhD}^{4,5}$
}

\begin{abstract}
Background: The role of hyaluronic acid plus hypertonic saline (HA+HS) as a mucoactive treatment in patients with bronchiectasis is still unknown. This study evaluated whether HA+HS solution enhances similar sputum quantity with better safety profile than HS alone in patients with bronchiectasis.

Methods: In this double-blind randomized crossover trial, three solutions (7\% HS; $0.1 \% \mathrm{HA}+7 \% \mathrm{HS}$; and $0.9 \%$ isotonic saline, IS) were compared in outpatients with bronchiectasis and chronic sputum expectoration. Participants inhaled each solution across four consecutive sessions. All sessions, except on session 3, also included 30 minutes of airway clearance technique. A 7-day washout period was applied. Sputum weight was collected during the sessions (primary outcome) as well as during a 24-hour follow-up. The Leicester Cough Questionnaire (LCQ) and lung function were measured before/after each treatment arm. Safety was assessed by the monitoring of adverse events (AEs).

Results: Twenty-eight patients with bronchiectasis (mean age of 64.0 (17.9) and $\mathrm{FEV}_{1} \% 60.9$ (24.6) of predicted) were recruited. HS and HA+HS promoted similar expectoration during sessions, both being greater than IS [median difference HS vs. IS $3.7 \mathrm{~g}$ (95\% CI 0.5-6.9); HA+HS vs. IS $3.2 \mathrm{~g}$ (95\% CI 0.5-5.9)]. Sputum expectorated exclusively during the ACT period was similar across all treatment arms [HS vs. IS $-0.3 \mathrm{~g}(95 \% \mathrm{CI}-1.7$ to 0.9$) ; \mathrm{HA}+\mathrm{HS}$ vs. IS $0.0 \mathrm{~g}$ (95\% CI-1.3 to 1.4); HS vs. HA+HS $0.0 \mathrm{~g}(95 \% \mathrm{CI}-1.2$ to 0.4)]. Sputum collected over the 24-hour follow-up tended to be lower for HS and HA+HS compared with IS [HS vs. IS - $1.7 \mathrm{~g}(95 \% \mathrm{CI}-4.2$ to 0.0$)$; HA+HS vs. IS - $1.1 \mathrm{~g}$ (95\%CI -3.6 to 0.7)]. No differences in LCQ or lung function were observed. Most severe AEs were reported using HS.

Conclusion: HS and HA+HS were more effective on sputum expectoration than IS in patients with bronchiectasis, reporting HA+HS better safety profile than HS.
\end{abstract}

Keywords: airway clearance techniques, bronchiectasis, hypertonic solutions, mucoactive treatment, sputum expectoration

\section{Introduction}

A CCORDING TO THE RECENT EUROPEAN GUIDELINES, the hyperosmolar agents plus airway clearance techniques (ACTs) may be considered to further enhance sputum expec- toration in adult patients with noncystic fibrosis bronchiectasis (henceforth referred to as "bronchiectasis" (1)); however, scientific evidence supporting this statement is quite scarce. ${ }^{(2)}$

The inhalation of hypertonic saline (HS) produces an osmotic shock in the airway surface layer that improves

\footnotetext{
${ }^{1}$ Universidad San Jorge, Zaragoza, Spain.

${ }^{2}$ Fundaciò Clínic, Hospital Clínic de Barcelona, Universitat de Barcelona, IDIBAPS, CIBERES, Barcelona, España.

${ }^{3}$ Facultat de Ciències de la Salut Blanquerna, Universitat Ramon Llull, Grup de Recerca en Salut, Activitat Física i Esport (SAFE), Barcelona, Spain.

${ }^{4}$ Servei de Pneumologia, Hospital Clínic de Barcelona, Universitat de Barcelona, IDIBAPS, CIBERES, Barcelona, España.

${ }_{5}^{5}$ Servei de Pneumologia, Hospital UniversitariValld'Hebron (HUVH), Institut de Recerca Valld'Hebron (VHIR), Barcelona, España.

(c) Beatriz Herrero-Cortina, et al., 2018. Published by Mary Ann Liebert, Inc. This Open Access article is distributed under the terms of the Creative Commons License (http://creativecommons.org/licenses/by/4.0), which permits unrestricted use, distribution, and reproduction in any medium, provided the original work is properly credited.
} 
airway hydration, accelerating mucus transportability. ${ }^{(3)}$ This effect of HS could potentiate and prolong the posterior effectiveness of ACTs. Greater sputum weight has been observed during combined sessions (inhalation and ACT) using HS rather than isotonic solution (IS) in patients with bronchiectasis and mild daily sputum expectoration $(<10 \mathrm{~g} /$ $24 \mathrm{~h}) .{ }^{(4)}$ However, the reasons why combined interventions (rather than individual) could be more efficient are unclear.

Overall, short and long-term use of HS has been demonstrated to be generally well tolerated by patients with bronchiectasis $^{(5)}$ and other respiratory diseases. ${ }^{(6,7)}$ Nevertheless, minor adverse events (AEs) (e.g., throat irritation, excessive coughing, or airway narrowing) have been frequently reported with HS inhalation. ${ }^{(8-10)}$ These minor AEs may appear after the first HS inhalation, negatively impacting long-term treatment adherence. ${ }^{(9,11)}$

Hyaluronic acid (HA) is a glycosaminoglycan that is able to mitigate bronchospasm induced by elastases and to balance water homeostasis in airways. ${ }^{(12,13)}$ Therefore, the addition of HA to HS (HA+HS) may be beneficial to improve tolerance and efficacy of HS solution. Previous studies conducted in cystic fibrosis showed greater tolerance and pleasantness in favor of $\mathrm{HA}+\mathrm{HS}$ compared with $\mathrm{HS}$ alone. ${ }^{(8,11,14)}$ So far, no studies have compared the effectiveness and tolerability of HA+HS and HS in adult outpatients with bronchiectasis. Improved tolerability with at least equal efficacy of HA+HS may improve patients' adherence, while hopefully improving chronic respiratory symptoms and quality of life (QoL) for patients with bronchiectasis.

Therefore, the purpose of this study was to evaluate whether $\mathrm{HA}+\mathrm{HS}$ is as efficacious as HS alone and better than IS in improving sputum expectoration (primary outcome), cough severity, and lung function in stable patients with bronchiectasis and daily expectoration ( $>10 \mathrm{~g} / 24 \mathrm{~h})$ naive to hyperosmolar agents. Also, this study aimed (i) to examine whether hypertonic solutions (HS, HA+HS) could increase effectiveness of ACTs in expectorated sputum compared with IS; (ii) to analyze whether a combined session of saline solutions and ACT is better than saline solutions alone in enhancing sputum quantity; (iii) to evaluate whether the short-term tolerability and safety of HA+HS is better compared with HS alone.

\section{Methods}

\section{Design}

A double-blind, randomized, crossover trial with concealed allocation was conducted. Participants each randomly inhaled one of the three solutions: HS solution (7\% $\mathrm{NaCl}) ; \mathrm{HA}+\mathrm{HS}$ solution $(0.1 \%$ sodium hyaluronate $+7 \%$ $\mathrm{NaCl})$, and IS solution $(0.9 \% \mathrm{NaCl})$, during four consecutive sessions (once daily). A 7-day washout period was applied between the treatment arms (Supplementary Fig. S1; Supplementary Fig. S1; Supplementary Data are available online at www.liebertpub.com/jamp).

A block random list was computer generated and retained by a research nurse, not directly involved in the project. The nurse received a notification email confirming the eligibility criteria by the enrolling investigator and the randomization took place after baseline data collection. Immediately, the sequence of treatments was revealed to the pharmacist (also external to the project) to produce the masking of inhaled solutions. Patients and physiotherapists were thus blinded to the inhaled solutions throughout the study.

The study was approved by the Hospital Clinic Research Ethics Committee (HCP/2011/6401) and was performed in accordance with the CONSORT statement (Clinical Trial Registration Number NCT02392663). All participants gave written informed consent before enrolment.

\section{Participants}

Patients were recruited from the Hospital Clinic, Barcelona, Spain. They were eligible to participate if over 18 years of age, diagnosed with bronchiectasis using highresolution computed tomography, clinically stable over the previous 4 weeks, ${ }^{(15)}$ producing spontaneous sputum expectoration (mean sputum $\geq 10 \mathrm{~g} / 24 \mathrm{~h}$ ), able to test the inhalation solutions, and to perform the ACT. The exclusion criteria included: smokers or former smokers $(\geq 10$-packyears), ${ }^{(16)}$ bronchial hyperresponsiveness diagnosis, ${ }^{(17)}$ asthma $^{(18)}$ or allergic bronchopulmonary aspergillosis, ${ }^{(5)}$ forced expiratory volume in the first second $\left(\mathrm{FEV}_{1}\right)<30 \%$ after bronchodilation, total lung capacity $<45 \%$, and inhalation of mucoactive agents before screening.

Finally, the withdrawal criteria were: pulmonary exacerbation during the study or any new medical/personal condition hindering study continuation.

\section{Intervention}

First, participants were encouraged to inhale $200 \mu \mathrm{g}$ of albuterol with a spacer chamber, or their usual bronchodilator, ${ }^{(19)}$ and before starting the inhalations they had to wait 15 minutes for short-acting bronchodilators or 30 minutes for long-acting bronchodilators. Participants inhaled $5 \mathrm{~mL}$ of the assigned solution through a mouthpiece using a jet nebulizer (PARI BOYSX ${ }^{\circledR}$ device with PARI LC ${ }^{\circledR}$ Sprint nebulizer) in a seated position. They were instructed and advised to inhale slowly and deeply follow by a short breath-hold (2-3 seconds) to improve aerosol deposition. ${ }^{(20)}$ Participants stopped compressor during coughing and all sessions were supervised by the physiotherapist to guarantee the correct mode of breathing during the inhalation.

All sessions for all treatment arms included 30 minutes of supervised ACT after inhalation, except for session 3. Autogenic drainage was the ACT chosen for the trial, based on our previous experience in terms of patient preference and short-term effectiveness ${ }^{(21)}$ and was performed in a supine position following the authors' recommendations. ${ }^{(22,23)}$ Physiotherapist gave oral advice and manual feedback during the performance. In each third session, participants remained 30 minutes in the same supine position after the inhalation period without performing any ACT ("control period").

Cough maneuvers were always spontaneous during sessions, and if necessary pauses were allowed. Peripheral oxygen saturation and heart rate were monitored during intervention. All study visits were performed to the same schedule at the hospital. Patients were asked not to perform ACTs before the beginning of the sessions and for 24 hours after the intervention.

Pharmacological treatments remained unchanged and patients were encouraged to take their long-term medications at the same time of the day over the study period. All 
participants were trained to breathe with the glottis open and coughing correctly before starting the trial.

\section{Outcome measures}

The primary outcome was the wet sputum expectoration $(g)$ collected during sessions. It was measured through two preweighed containers, one to collect the sputum expectorated during inhalation period and the other to measure the sputum collected during ACT period. For the primary outcome, session 3 (inhalation period + control period) was not included, but the sputum expectorated was also measured following the same procedure.

Secondary endpoints included the spontaneous sputum expectorated over a 24-hour follow-up after the end of sessions, collected in another preweighed container. The timeline chosen for evaluating the long-lasting effects of interventions was based on similar previous studies on sputum expectoration. ${ }^{(21,24,25)}$

Secretions from sinus after an inspiratory forced maneuver were not allowed to include in the containers. Participants were instructed to swallow saliva before coughing during inhalation periods and also to avoid salivary contamination over a 24-hour follow-up after intervention. In addition, most part of possible saliva was removed from the containers before weighing.

Cough severity, using the Leicester Cough Questionnaire $(\mathrm{LCQ})^{(26,27)}$ and lung function $\left(\mathrm{FEV}_{1}\right.$ and $\left.\mathrm{FVC}\right),{ }^{(28)}$ was also assessed at the beginning and end of each treatment arm. At the end of the trial, participants selected their preferred treatment arm while also determining which solution was the saltiest.

Safety was assessed by monitoring AEs after each inhalation. ${ }^{(11)}$ Perception of bronchospasm (wheezing or chest tightness), excessive coughing, and throat irritation were reported by participants and their severity was measured using a three-point ordinal score ${ }^{(11)}(0=$ absent, $1=$ mild, $2=$ moderate, and $3=$ severe; from 0 to 9). The presence of hemoptysis and desaturation was assessed by the physiotherapist.

Tolerability was evaluated the first day of each saline solution tested. A spirometry was performed pre- and postbronchodilator and again 5 minutes after completing the inhalation. ${ }^{(29)}$ Participants with inhalation-induced bronchospasm (fall $\left.\geq 12 \% \mathrm{FEV}_{1}\right)^{(30)}$ were not allowed to continue the remainder sessions of the same treatment arm; however, they continued the study trying the next saline solution according to the same schedule and procedure.

\section{Data analysis}

A sample size of 20 completing patients was calculated to be necessary to provide $80 \%$ power and $5 \%$ level of significance, in a two-sided test, to detect a minimum $5.7 \mathrm{~g}^{(21)}$ of difference in sputum quantity during sessions, including inhaled and ACT periods, between the three treatment arms. This is based on the fact that the standard deviation of difference in the response variable for the same patient is 8.5. ${ }^{(21)}$ Allowing for $20 \%$ early withdrawal, this study recruited a total of 24 patients.

Repeated-measures analysis was performed using linear mixed models to determine changes in expectorated sputum, cough severity, lung function, and safety score across the three treatment arms. Allocation sequence, group, and treatment were considered as fixed effects, and subjects within sequence were considered a random effect. The daily expectoration measured before starting each treatment period was incorporated into the model as a covariance (except for safety analysis). Difference between the treatment arms was reported as median difference (95\% Confidence Interval $[\mathrm{CI}])^{(31)}$ and statistical significance was set at $p<0.05$ for all calculations.

All randomized participants were included in the analysis for effectiveness (intention-to-treat basis); however, those who never began treatment after the randomization process (dropouts before the first session), did not complete the study, or pass the tolerability test for all solutions, were not included in the safety analysis. ${ }^{(32)}$ Missing data were not imputed. ${ }^{(33,34)}$

The sputum collected during the intervention and over the 24-hour follow-up was compared with a combined session, including ACT period (session 1, 2, or 4), and the single session, including a control period (session 3) using a Wilcoxon test for each of the treatment arms. To select only one of the three possible ACT sessions, a list of random numbers was generated. The sputum obtained was analyzed as sputum weight and sputum quantity ratio (\%) (i.e., sputum expectorated during the session or during the 24-hour follow-up/total sputum collected $\times 100$ ). Effect sizes were computed to estimate the magnitude of changes, using rankbiserial correlation $(r)$ and interpreted as small effect $(<0.3)$, moderate effect $(\geq 0.3)$, and large effect $(\geq 0.5)$.

\section{Results}

From March to December 2015, thirty-eight volunteers were screened for the study, with 28 meeting the eligibility criteria, who were consented and randomized. Three participants dropped out of the study before the first session and two participants withdrew during the study (Supplementary Fig. S2). Accordingly, 23 patients completed the study protocol with $98 \%$ adherence to planned treatment sessions.

Participant characteristics are shown in Table 1. At the beginning of all treatment arms, participants were similar with respect to daily sputum expectoration, total LCQ score, and lung function, indicating no carryover effects.

\section{Treatment effect}

Sputum expectoration. The primary outcome, sputum collected during sessions (including inhalation plus ACT periods) with HS and $\mathrm{HA}+\mathrm{HS}$ treatments, was similar, both being greater than that obtained with IS (Table 2).

Similarly, the sputum obtained exclusively during the inhalation period was also similar to the HS and HA+HS treatments, both being greater than that obtained with IS. However, sputum expectorated exclusively during the ACT period was similar across all treatment arms. The sputum collected over the 24-hour follow-up showed a decreasing trend from IS (the highest), to HA+HS and HS, being the effects of hypertonic solutions being very similar (Table 2).

When comparing the random combined session, including ACT (session 1, 2 or 4) and that without it (session 3), we consistently observed more sputum in the combined session at the end of sessions, independently of the inhaled solution (Table 3). Consequently, the session sputum quantity ratio (\%) obtained was always greater for the combined session 
Table 1. Baseline Characteristics of RANDOMIZEd Participants

Characteristics $(\mathrm{n}=28)$

Age (yr), mean (SD)

Gender, $n$ female $(\%)$

BMI $\left(\mathrm{kg} / \mathrm{m}^{2}\right)$, mean (SD)

$64.0(17.5)$

$18(64.3)$

Etiology of bronchiectasis, $n(\%)$

Unknown

$24.3(3.6)$

Postinfection

$10(35.7)$

Primary ciliary dyskinesia

Others

Chronic airway infection $(\%)^{\mathrm{a}}$

$P$. aeruginosa infection

$12(42.8)$

$3(10.7)$

$3(10.7)$

$22(78.6)$

$14(50)$

Lung function $\left(\mathrm{FEV}_{1}\right)$

Liters

$1.5(0.8)$

$\%$ pred.

$60.9(24.6)$

Long-term inhaled $\beta_{2}$ agonists, $n(\%)$

Short acting

Long acting

Long-term inhaled anticholinergics, $n(\%)$

Short acting

Long acting

Long-term inhaled steroid therapy, $n(\%)$

Long-term antibiotic treatment, $n(\%)$

Oral (macrolides)

Inhaled

Baseline sputum expectoration $^{\mathrm{b}}$

24-hour period, median (IQR)

$3(10.7)$

$22(76.6)$

$2(7.1)$

$14(50.0)$

$21(75.0)$

$3(10.7)$

$3(10.7)$

$13.6(10.8-21.4)$

Data presented as $n, n(\%)$, mean (SD, standard deviation) and median (IQR, interquartile range).

${ }^{a}$ Chronic airway infection was defined as pathogen organism cultured in at least two or more sputum samples, at least 3 months apart, in the preceding 12 months.

${ }^{\mathrm{b}}$ Measured on two consecutive days during the week before the start of the study.

BMI, body mass index; FEV1, forced expiratory volume in 1 second; FVC, forced vital capacity, \% pred, percentage of predicted.

than session 3 [HS Inh+ACT vs. Inh+Control $14.5 \%$ (95\% CI 6.2-24.5); HA+HS Inh+ACT vs. Inh+Control $11.4 \%$ (95\% CI 2.2-20.3); IS Inh+ACT vs. Inh+Control $24.1 \%$ (95\% CI 11.1-35.9)] (Fig. 1).

On the contrary, the sputum quantity ratio obtained during the 24-hour follow-up period was consistently proportionally lower in the random combined session, including ACT (session 1 or 2 or 4 ), than in session 3 , including control period for all solutions [HS Inh+ACT vs. Inh+Control $-11.6 \%$ (95\% CI -22.9 to -3.2$)$; HA+HS Inh+ACT vs. In$h+$ Control $-9.4 \%$ (95\% CI -19.3 to -1.7$)$; IS Inh+ACT vs. Inh+Control $-20.7 \%$ (95\% CI -34.4 to -8.6)] (Fig. 1).

Finally, the time spent during inhalation period was similar between the three solutions $(p=0.06)$, showing greater amounts of time spent with HS (20 minutes and 58 seconds) and HA+HS (18 minutes and 39 seconds) treatments compared with IS (16 minutes and 5 seconds) treatment.

Cough severity and lung function. By comparing the 3 treatments, the observed changes in total LCQ were not significantly different $(p>0.05)$. Lung function $\left(\mathrm{FEV}_{1}\right.$ and FVC) also remained unchanged after all interventions $(p>0.05)$ (Supplementary Table S1).

Preference and salty taste. Around $48 \%$ of participants who started all treatment arms selected HA+HS as their preferred solution to include in chronic treatment. As expected, most of the patients (69\%) reported HS as the saltiest solution.

\section{Safety and tolerability of the intervention}

HS showed the poorest safety profile [HS vs. IS 2.7 (1.63.9); HS vs. HA+HS 1.2 (0.3-2.4)]; whereas HA+HS showed an intermediate safety profile between HS and IS [HA+HS vs. IS $1.2(0.3-2.0)$ ] (Supplementary Table S2). Coughing and throat irritation were the most frequent minor AEs classified as moderate or severe by participants, particularly after the inhalation of HS and, to a lesser extent, after HA+HS (Fig. 2). Mild oxygen desaturation was detected in three patients, but the values increased to normal after the inhalation period. Three small sputum samples stained with blood from two participants (one during IS solution and the other during HS and IS solution) were observed.

While IS solution was well tolerated by all participants, the tolerability test of the HS solution failed in three patients. Two of these three patients also failed the tolerability test for the HA+HS solution (Supplementary Fig. S2). These three participants were elderly men ( $\geq 75$ years) and presented a major impairment of lung function $\left(\mathrm{FEV}_{1} \leq 40 \%\right.$ of predicted) compared with the sample average.

Consequently, the three treatment arms were completed from $71 \%$ of randomized participants. Individually, 20

Table 2. Median (Interquartile Range) Values of Sputum Expectorated during Sessions 1,2 , and 4 at Different Time Points for Each Treatment Arm and Median Difference (95\% CI) Between Treatment Arms

\begin{tabular}{|c|c|c|c|c|c|c|}
\hline & \multicolumn{3}{|c|}{ Treatment arms $(\mathrm{n}=28)$} & \multicolumn{3}{|c|}{ Median difference between treatment arms $(\mathrm{n}=28)$} \\
\hline & $H S$ & $H A+H S$ & IS & $H S$ vs. $H A+H S$ & $H S$ vs. $I S$ & $H A+H S$ vs. IS \\
\hline Inhalation $+\mathrm{ACT}^{\mathrm{a}}$ & $11.3(0.4-19.8)$ & $10.7(4.2-18.8)$ & $8.4(2.9-15.3)$ & $0.4(-0.4$ to 2.2$)$ & $3.7(0.5-6.9)$ & $3.2(0.5-5.9)$ \\
\hline Inhalation & $6.6(0.4-11.2)$ & $5.8(0.9-11.1)$ & $3.0(0.9-5.2)$ & $1.6(0.0-2.8)$ & $4.0(1.6-6.7)$ & $2.6(1.3-4.6)$ \\
\hline ACT & $4.0(0.0-7.8)$ & $3.9(1.3-9.6)$ & $5.4(1.7-8.4)$ & $0.0(-1.2$ to 0.4$)$ & $-0.3(-1.7$ to 0.9$)$ & $0.0(-1.3$ to 1.4$)$ \\
\hline 24-hour follow-up ${ }^{b}$ & $4.8(0.4-11.0)$ & $7.7(2.7-10.4)$ & $8.4(3.6-12.6)$ & $-0.2(-2.2$ to 1.4$)$ & $-1.7(-4.2$ to 0.0$)$ & $-1.1(-3.6$ to 0.7$)$ \\
\hline
\end{tabular}

The amount of sputum was measured in grams $(\mathrm{g})$.

${ }^{\text {a Primary outcome. }}$

${ }^{\mathrm{b}}$ Twenty-four hour follow-up does not include the time of intervention. Comparisons were adjusted by the level of expectoration collected before starting each treatment arm.

HS, hypertonic saline; HA + HS, hyaluronate acid plus hypertonic saline; IS, isotonic saline; ACT, airway clearance technique. 
Table 3. Median (Interquartile Range) of Sputum Expectorated During the Random Combined Session, Including ACT Period (Session 1 or 2 or 4) And the Single Session, Including Control Period (Session 3) at the Different Periods for Each One of the Treatment Arms, Median Difference (95\% CI) Between the Sessions

\begin{tabular}{|c|c|c|c|c|}
\hline & \multicolumn{2}{|c|}{ Treatment arms $(\mathrm{n}=28)$} & \multirow{2}{*}{$\begin{array}{c}\text { Median difference }(95 \% \text { CI }) \\
\text { Session including ACT vs. Session } \\
\text { including control period }\end{array}$} & \multirow[b]{2}{*}{ Effect size } \\
\hline & $\begin{array}{l}\text { Session including } \\
\text { ACT period }\end{array}$ & $\begin{array}{l}\text { Session including } \\
\text { control period }\end{array}$ & & \\
\hline \multicolumn{5}{|l|}{ HS } \\
\hline Inhalation $+\mathrm{ACT} /$ control & $12.1(0.5-21.8)$ & $6.3(0.0-11.7)$ & $4.5(2.1-9.7)$ & 0.61 \\
\hline Inhalation & $5.3(0.5-12.7)$ & $5.4(0.2-9.4)$ & $1.0(0.0-3.0)$ & 0.37 \\
\hline ACT/control & $4.6(0.0-7.5)$ & $0.0(0.0-2.0)$ & $3.3(1.8-5.4)$ & 0.68 \\
\hline 24-hour follow-up ${ }^{\mathrm{a}}$ & $3.4(0.0-12.7)$ & $7.8(0.0-19.7)$ & $-2.7(-7.5$ to -0.3$)$ & 0.57 \\
\hline \multicolumn{5}{|l|}{$\mathrm{HA}+\mathrm{HS}$} \\
\hline Inhalation $+\mathrm{ACT} /$ control & $11.5(3.2-18.4)$ & $6.2(0.0-13.3)$ & $4.0(0.8-6.6)$ & 0.52 \\
\hline Inhalation & $6.3(0.8-11.4)$ & $4.5(0.3-11.4)$ & $0.0(-2.0$ to 2.3$)$ & 0.00 \\
\hline ACT/control & $4.0(0.8-8.7)$ & $0.0(0.0-1.9)$ & $2.9(1.7-5.3)$ & 0.67 \\
\hline 24-hours follow-up ${ }^{a}$ & $7.0(1.6-12.5)$ & $8.1(2.3-18.9)$ & $-2.1(-6.4$ to -0.3$)$ & 0.53 \\
\hline \multicolumn{5}{|l|}{ IS } \\
\hline Inhalation $+\mathrm{ACT} /$ control & $8.6(3.4-13.7)$ & $3.0(0.0-4.7)$ & $5.1(3.5-8.4)$ & 0.72 \\
\hline Inhalation & $2.0(0.1-4.6)$ & $1.6(0.0-3.5)$ & $0.5(-0.5$ to 1.3$)$ & 0.16 \\
\hline ACT/control & $5.7(1.6-8.3)$ & $0.5(0.0-2.0)$ & $4.4(2.7-6.9)$ & 0.76 \\
\hline 24-hour follow-up ${ }^{a}$ & $6.0(2.1-13.1)$ & $10.0(5.4-17.8)$ & $-1.9(-6.6$ to -0.1$)$ & 0.38 \\
\hline
\end{tabular}

The amount of sputum was measured in grams $(\mathrm{g})$.

${ }^{a}$ Twenty-four hour follow-up does not include the time of intervention.

(71\%) patients completed the HS solution arm, $22(79 \%)$ the $\mathrm{HA}+\mathrm{HS}$ solution arm, and $23(82 \%)$ the IS solution arm.

\section{Discussion}

This is the first study to evaluate the impact of three different saline solutions (HS, HA+HS, IS) inhaled before ACT on sputum expectoration in adult outpatients with bronchiectasis and chronic expectoration $(>10 \mathrm{~g} / 24 \mathrm{~h})$.

The main findings were: (i) both hypertonic solutions at $7 \%$ (HS and $\mathrm{HA}+\mathrm{HS}$ ) promoted greater sputum weight during sessions than IS. Contrarily, the sputum collected over the 24-hour follow-up after sessions showed a de- creasing trend from IS to HA+HS and, finally, to HS (the lowest). No significant changes in cough severity and lung function were observed between the treatment arms after four sessions; (ii) effectiveness of ACT (using autogenic drainage) seemed not to be influenced by the previously inhaled solutions; in fact the sputum obtained during the ACT period was similar throughout all treatment arms; (iii) sputum collected at the end of a combined session (inhaled + ACT period) was always greater than a single session, including the control period (session 3), independently of the inhaled solution; conversely, the sputum expectorated in the combined sessions 24 hours hence was clearly lower than after session 3 (inhalation + control); (iv) globally, the

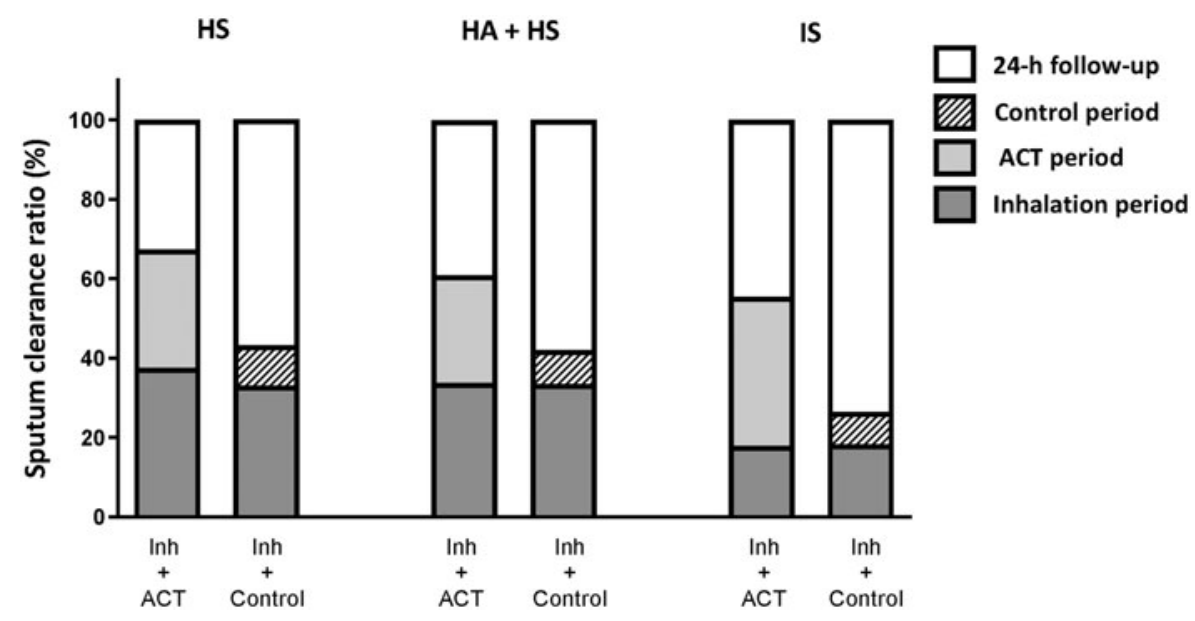

FIG. 1. Percentage $(\%)$ of sputum clearance collected at different time points during the random combined session (day 1 or 2 or 4), including ACT period and the single session (session 3), including control period. ACT, airway clearance technique; HS, hypertonic saline; HA+HS, hyaluronate acid plus hypertonic saline; IS, isotonic saline. 

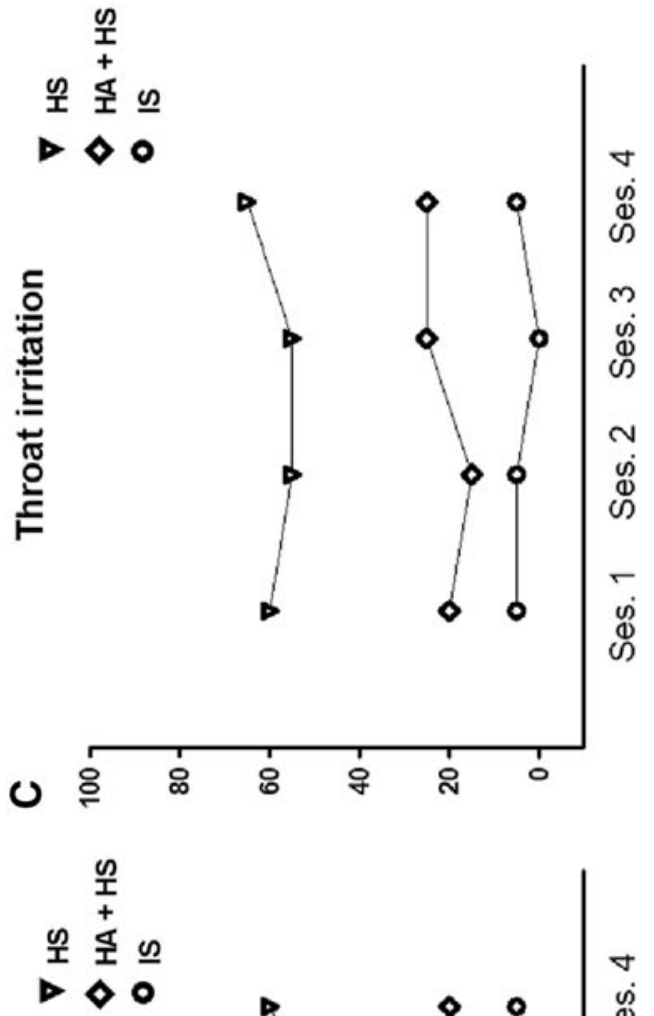

들

m
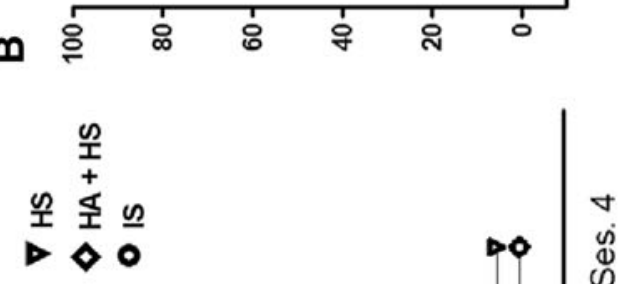

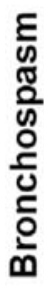

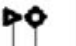

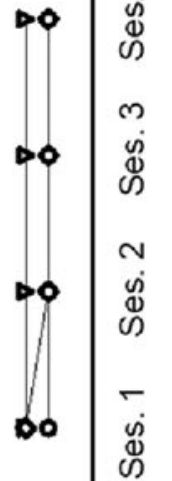

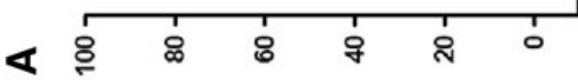

solutions were well tolerated, but AEs during inhalation were more frequent and severe, from HS to $\mathrm{HA}+\mathrm{HS}$ to IS (lowest).

The effect duration of hypertonic solutions (HS and $\mathrm{HA}+\mathrm{HS}$ ) is poorly known in bronchiectasis, but their shortterm efficacy may be related to their improved biophysical sputum properties (lower adhesivity and greater cough transportability) ${ }^{(35,36)}$ The major short-term efficacy of hypertonic solutions was also confirmed by the lower sputum expectoration observed in the 24 hours following HS and $\mathrm{HA}+\mathrm{HS}$, compared with IS. Hypertonic solutions were able to concentrate greater amounts of sputum during the treatment period and reduce the need to expectorate throughout the rest of the day, being one of the main short-term goals of airway clearance treatment. ${ }^{(37)}$ A similar finding was described during the 24-hour period following the intervention in a previous trial comparing different ACTs in patients with bronchiectasis. ${ }^{(21)}$

However, this result should be taken with caution because the measure of sputum expectoration has some potential limitations (saliva contamination, involuntary swallowing, patient compliance) and does not necessarily reflect the impact on airway clearance.

Kellet et al. ${ }^{(4)}$ did not clearly describe whether the increased sputum weight obtained using HS compared with IS was due to the osmolality of the different solutions or to the increased effectiveness of ACT after the inhalation of HS in patients with bronchiectasis and mild daily sputum expectoration $(<10 \mathrm{~g} / 24 \mathrm{~h})$. In our study, no increased effectiveness during ACT was observed following hypertonic solutions, despite their higher osmotic impact on the airway surface layer compared with IS. Baseline sputum expectoration in our population ( $>10 \mathrm{~g} / 24 \mathrm{~h}$ ) was greater compared with Kellet' study, thus proving to be irrelevant to the efficacy of hyperosmolar solutions. Moreover, our findings confirm that the advantage of $\mathrm{HS}$ and $\mathrm{HA}+\mathrm{HS}$ on sputum expectoration is more related to the inhalation than to ACT.

Unfortunately, the study design did not have a single ACT arm (no previous inhalations) that would have helped to better understand the interaction between inhalations and the efficacy of ACT. Thus, future studies are recommended to further investigate if the efficacy of ACTs is influenced by previous saline solutions in bronchiectasis.

Similarly, inhalation of HA alone was not evaluated in the present study. Previous studies suggest that inhalation of HA reduces elastin degradation in COPD and prevents bronchoconstriction in people with asthma without AEs reported. $^{(13,38,39)}$ To the authors' best knowledge, inhalation of HA alone has never been explored in bronchiectasis and further research is needed to evaluate the impact of this treatment on mucus clearance and airway inflammation.

Participants used bronchodilators before all inhalations to improve tolerability, ${ }^{(2)}$ optimize pulmonary deposition, ${ }^{(2)}$ and improve cough clearance of secretions by increasing expiratory flow. ${ }^{(40)}$ It is known that $\beta$-agonist bronchodilators may stimulate ciliary beat frequency and anticholinergics can decrease volume of secretions that is triggered by airway inflammation. ${ }^{(41)}$ In this study, participants were clinically stable throughout the study, their medication was unchanged, and the study design allowed intrasubject comparison, ${ }^{42)}$ therefore the impact of bronchodilators on sputum expectoration differences between treatment arms is limited. 
The length of combined sessions (inhalation + ACT period) may be considered burdensome by patients. Considering our results, it might be recommended to include an ACT after inhalation to achieve a greater reduction of daily expectoration after intervention, independently of the solution previously inhaled. Studies conducted in patients with cystic fibrosis suggest that the timing of HS (before or during ACTs) had no impact on the clinical effectiveness, ${ }^{(43,44)}$ although it clearly reduces the time burden associated with treatment and may promote future adherence. Future studies are required to explore if $\mathrm{HS}$ and $\mathrm{HA}+\mathrm{HS}$ inhalation during ACT has similar results in patients with bronchiectasis as well as an equal reduction in the need for expectoration after the intervention.

Previous studies comparing the effects of HS and IS on QoL and lung function have demonstrated controversial findings. ${ }^{(5)}$ No significant changes were observed in LCQ score and lung function across all treatment arms. Perhaps the short duration of our intervention did not enable us to observe a significant impact on the LCQ, a subjective assessment of cough. Thus, future studies may incorporate cough monitoring to detect objectively differences in cough frequency between interventions. ${ }^{(45)}$

Furthermore, it has been hypothesized that the response to hypertonic saline could increase with the severity of lung function impairment. ${ }^{(3)}$ Therefore, it is likely that greater changes in cough severity and lung function could be expected in patients with more severe bronchiectasis and lung function impairment, unlike our population which showed a moderate lung obstruction $\left(\mathrm{FEV}_{1}=61 \%\right.$ of predicted).

Globally, the saline solutions were well tolerated. The participants that did not pass the tolerability test for hypertonic solutions were elderly with severe lung obstruction. As expected, the main AEs reported were with HS, whereas $\mathrm{HA}+\mathrm{HS}$ showed an improved safety profile, between those of HS and IS. These findings agree with previous studies conducted within cystic fibrosis, ${ }^{(8,11,14)}$ and support the alternative use of $\mathrm{HA}+\mathrm{HS}$ in bronchiectasis. The relatively low rate of AEs of HA+HS may also explain why it was chosen as the preferred saline solution by patients.

The present study has different strengths: despite the weighing wet sputum being controversial as an outcome measure ${ }^{(46)}$ and only can be interpretable over a short-effect period, ${ }^{(37)}$ the crossover study design with consecutive sessions improved the accuracy of our results, allowing intrasubject comparisons. ${ }^{(42)}$ The repeated measurements of sputum at different points in time (inhalation, ACT, and 24 hours follow-up) also better describe immediate and shortterm effects on sputum expectoration, such as frequency and severity of the AEs of the different saline solutions in patients with bronchiectasis. For future long-term trials, mucus dehydration (e.g., sputum percentage solids) or sputum inflammatory cells may be a useful measurement to assess the effect of hypertonic saline solutions on disease severity.

The main limitation was that most participants identified the HS solution by its salty taste; however, HA+HS and IS were not easily identifiable. It was decided not to use quinine sulfate as a blinding agent, as it is unknown whether it may influence the HA+HS properties and alter its effectiveness. However, our participants were naive to inhaled saline solutions and not informed about the different characteristics of the three treatment arms. A similar limitation was observed in the Nicolson et al. ${ }^{(47)}$ study, demonstrating that salty taste does not consistently affect the blindness of participants.

Finally, the high dropout rate throughout the study may be another limitation. Nevertheless, the primary reason for withdrawal was related to the time burden perceived by participants. Although the research protocol could have been easily performed at home, we preferred the hospital setting to guarantee a close monitoring of AEs.

In conclusion, we found that inhaled $\mathrm{HA}+\mathrm{HS}$ was as efficacious as HS in improving sputum expectoration, but with a better safety profile in patients with bronchiectasis. Moreover, the additional performance of ACT (autogenic drainage) after hypertonic solutions achieved the greater sputum weight, and a significant reduction in expectoration for the rest of the day. The daily use of hypertonic saline solutions, combined with ACT, may be useful in reducing the burden of daily symptoms, although further investigation is needed to assess long-term outcomes.

\section{Acknowledgments}

The authors are grateful to the participants and the hospital staff for their assistance in conducting the trial, particularly to Rebeca Domingo and Montserrat Solá.

This project was sponsored by Praxis Pharmaceuticals and Chiesi Farmaceutici, providing the study drug (hyaluronate acid + hypertonic saline solutions). Funding sources had no involvement in the study concept or design; in the collection, analysis, or interpretation of data; in the writing of the report or in the decision to submit the article for publication.

\section{Authors' Contribution}

BH-C was directly involved in the project design, study development, data collection, statistical analysis, and article draft. V-A contributed to data collection and article draft. JV and AT contributed to data interpretation and to critical revision of the article for intellectual concept. EP led the study, being responsible for the overall quality and content of the article. All authors have reviewed and approved the final version of the article.

\section{Author Disclosure Statement}

The authors declared no potential conflicts of interest with respect to the research, authorship, and/or publication of this article.

\section{References}

1. Chalmers JD and Elborn JS: Reclaiming the name "bronchiectasis'. Thorax. 2015;70:399-400.

2. Polverino E, Goeminne PC, McDonnell MJ, Aliberti S, Marshall SE, Loebinger MR, Murris M, Cantón R, Torres A, Dimakou K, De Soyza A, Hill AT, Haworth CS, Vendrell M, Ringshausen FC, Subotic D, Wilson R, Vilaró J, Stallberg B, Welte T, Rohde G, Blasi F, Elborn S, Almagro M, Timothy A, Ruddy T, Tonia T, Rigau D, and Chalmers JD: European Respiratory Society guidelines for the management of adult bronchiectasis. Eur Respir J. 2017;50: 1700629. 
3. Bennett WD, Henderson AG, and Donaldson SH: Hydrator Therapies for Chronic Bronchitis. Lessons from Cystic Fibrosis. Ann Am Thorac Soc. 2016;13 Suppl 2:S186S190.

4. Kellett F, Redfern J, and Niven RM: Evaluation of nebulised hypertonic saline (7\%) as an adjunct to physiotherapy in patients with stable bronchiectasis. Respir Med. 2005;99:27-31.

5. Hart A, Sugumar K, Milan SJ, Fowler SJ, and Crossingham I: Inhaled hyperosmolar agents for bronchiectasis. Cochrane Database Syst Rev. 2014;5:CD002996.

6. Alexis NE, Bennett W, and Peden DB: Safety and benefits of inhaled hypertonic saline following airway challenges with endotoxin and allergen in asthmatics. J Asthma. 2017; 54:957-960.

7. Donaldson SH, Bennett WD, Zeman KL, Knowles MR, Tarran R, and Boucher RC: Mucus clearance and lung function in cystic fibrosis with hypertonic saline. N Engl J Med. 2006;354:241-250.

8. Ros M, Casciaro R, Lucca F, Troiani P, Salonini E, Favilli F, Quattrucci S, Sher D, and Assael BM: Hyaluronic acid improves the tolerability of hypertonic saline in the chronic treatment of cystic fibrosis patients: A multicenter, randomized, controlled clinical trial. J Aerosol Med Pulm Drug Deliv. 2014;27:133-137.

9. Elkins MR, Robinson M, Rose BR, Harbour C, Moriarty CP, Marks GB, Belousova EG, Xuan W, Bye PT, and Group NHSiCFNS. A controlled trial of long-term inhaled hypertonic saline in patients with cystic fibrosis. $\mathrm{N}$ Engl J Med. 2006;354:229-240.

10. Valderramas SR and Atallah AN: Effectiveness and safety of hypertonic saline inhalation combined with exercise training in patients with chronic obstructive pulmonary disease: A randomized trial. Respir Care. 2009;54:327333.

11. Buonpensiero P, De Gregorio F, Sepe A, Di Pasqua A, Ferri P, Siano M, Terlizzi V, and Raia V: Hyaluronic acid improves "pleasantness" and tolerability of nebulized hypertonic saline in a cohort of patients with cystic fibrosis. Adv Ther. 2010;27:870-878.

12. Brivio A, Conese M, Gambazza S, Biffi A, Tirelli AS, Russo $\mathrm{M}$, Foà $\mathrm{M}$, and Colombo $\mathrm{C}$ : Pilot randomized controlled trial evaluating the effect of hypertonic saline with and without hyaluronic acid in reducing inflammation in cystic fibrosis. J Aerosol Med Pulm Drug Deliv. 2016;29: 482-489.

13. Turino GM and Cantor JO: Hyaluronan in respiratory injury and repair. Am J Respir Crit Care Med. 2003;167: 1169-1175.

14. Cazzarolli C, Tartali C, and Pradal U: The advantages of adding hyaluronic acid or mannitol to hypertonic saline inhalation treatment in cystic fibrosis. J Aerosol Med Pulm Drug Deliv. 2017;30:141-142.

15. Murray MP, Govan JR, Doherty CJ, Simpson AJ, Wilkinson TS, Chalmers JD, Greening AP, Haslett C, and Hill AT: A randomized controlled trial of nebulized gentamicin in non-cystic fibrosis bronchiectasis. Am J Respir Crit Care Med. 2011;183:491-499.

16. Murray MP, Pentland JL, and Hill AT: A randomised crossover trial of chest physiotherapy in non-cystic fibrosis bronchiectasis. Eur Respir J. 2009;34:1086-1092.

17. Cockcroft DW and Davis BE: Mechanisms of airway hyperresponsiveness. J Allergy Clin Immunol. 2006;118:551559; quiz 560-561.
18. Global Initiative for Asthma. Global Strategy for Asthma Management and Prevention, 2018. Available from www. ginasthma.org, accessed March 15, 2018.

19. Dentice RL, Elkins MR, and Bye PT: Adults with cystic fibrosis prefer hypertonic saline before or during airway clearance techniques: A randomised crossover trial. J Physiother. 2012;58:33-40.

20. Orlik T and Sands D: Application of positive expiratory pressure *PEP* in cystic fibrosis patient inhalations. Dev Period Med. 2015;19:50-59.

21. Herrero-Cortina B, Vilaró J, Martí D, Torres A, San Miguel-Pagola M, Alcaraz V, and Polverino E: Short-term effects of three slow expiratory airway clearance techniques in patients with bronchiectasis: A randomised crossover trial. Physiotherapy. 2016;102:357-364.

22. Button BM and Button B: Structure and function of the mucus clearance system of the lung. Cold Spring Harb Perspect Med. 2013;3: pii: a009720.

23. Agostini $P$ and Knowles N: Autogenic drainage: The technique, physiological basis and evidence. Physiotherapy. 2007;93:157-163.

24. Munkholm M and Mortensen J: Mucociliary clearance: Pathophysiological aspects. Clin Physiol Funct Imaging. 2014;34:171-177.

25. Muñoz G, de Gracia J, Buxó M, Alvarez A, and Vendrell M: Long-term benefits of airway clearance in bronchiectasis: A randomised placebo-controlled trial. Eur Respir J. 2018;51: pii: 1701926.

26. Muñoz G, Buxó M, de Gracia J, Olveira C, MartinezGarcia MA, Giron R, Polverino E, Alvarez A, Birring SS, and Vendrell M: Validation of a Spanish version of the Leicester Cough Questionnaire in non-cystic fibrosis bronchiectasis. Chron Respir Dis. 2016;13:128-136.

27. Raj AA, Pavord DI, and Birring SS: Clinical cough IV: what is the minimal important difference for the Leicester Cough Questionnaire? Handb Exp Pharmacol. 2009;187: 311-320.

28. Miller MR, Hankinson J, Brusasco V, Burgos F, Casaburi $\mathrm{R}$, Coates A, Crapo R, Enright P, van der Grinten CP, Gustafsson P, Jensen R, Johnson DC, MacIntyre N, McKay R, Navajas D, Pedersen OF, Pellegrino R, Viegi G, Wanger $\mathrm{J}$, and Force AET: Standardisation of spirometry. Eur Respir J. 2005;26:319-338.

29. Pasteur MC, Bilton D, Hill AT, and Group BTSBn-CG. British Thoracic Society guideline for non-CF bronchiectasis. Thorax. 2010;65 Suppl 1:i1-i58.

30. Pradal U, Borruso A, Saloni E, Fedrigo E, Menin L, Tartali $\mathrm{C}$, and Assael BM: Hyaluronic acid in the prevention of bronchial obstruction induced by hypertonic saline J Cyst Fibros. 2010;9(Suppl 1):S62.

31. Campbell MJ and Gardner M: Medians and their differences. In: Statistics with Confidence, Second edition. Altman D, Machin D, Bryant T, and Gardner M (eds). BMJ books, Bristol, UK. 2000.

32. Ioannidis JP, Evans SJ, Gøtzsche PC, O’Neill RT, Altman DG, Schulz K, Moher D, and Group C: Better reporting of harms in randomized trials: An extension of the CONSORT statement. Ann Intern Med. 2004;141:781-788.

33. Twisk J, de Boer M, de Vente W, and Heymans M: Multiple imputation of missing values was not necessary before performing a longitudinal mixed-model analysis. J Clin Epidemiol. 2013;66:1022-1028.

34. Rosenkranz GK: Analysis of cross-over studies with missing data. Stat Methods Med Res. 2015;24:420-433. 
35. Redding GJ, Kishioka C, Martinez P, and Rubin BK: Physical and transport properties of sputum from children with idiopathic bronchiectasis. Chest. 2008;134:11291134.

36. Rubin BK: The role of mucus in cough research. Lung. 2010;188 Suppl 1:S69-S72.

37. Bradley J, O’Neill K, Vilaro J, and Mcllwaine M: Airway clearance in bronchiectasis. In: J Chalmers, E Polverino, and S Aliberti, (eds). Bronchiectasis. The EMBARC manual.; pp. 257-284, 2018.

38. Turino GM, Ma S, Lin YY, and Cantor JO: The therapeutic potential of hyaluronan in COPD. Chest. 2018;153:792798.

39. Cantor J, Ma S, and Turino G: A pilot clinical trial to determine the safety and efficacy of aerosolized hyaluronan as a treatment for COPD. Int J Chron Obstruct Pulmon Dis. 2017;12:2747-2752.

40. Rubin BK: Secretion properties, clearance, and therapy in airway disease. Transl Respir Med. 2014;2:6.

41. Rubin BK: Aerosol medications for treatment of mucus clearance disorders. Respir Care. 2015;60:825-829; discussion 830-832.

42. Mills EJ, Chan AW, Wu P, Vail A, Guyatt GH, and Altman DG: Design, analysis, and presentation of crossover trials. Trials. 2009;10:27.

43. Elkins M and Dentice R: Timing of hypertonic saline inhalation for cystic fibrosis. Cochrane Database Syst Rev. 2016;12:CD008816.

44. O’Neill K, Moran F, Tunney MM, Elborn JS, Bradbury I, Downey DG, Rendall J, and Bradley JM: Timing of hypertonic saline and airway clearance techniques in adults with cystic fibrosis during pulmonary exacerbation: Pilot data from a randomised crossover study. BMJ Open Respir Res. 2017;4:e000168.

45. Spinou A, Lee KK, Sinha A, Elston C, Loebinger MR, Wilson R, Chung KF, Yousaf N, Pavord ID, Matos S, Garrod R, and Birring SS: The Objective Assessment of Cough Frequency in Bronchiectasis. Lung. 2017;195:575-585.

46. Rubin BK: Designing clinical trials to evaluate mucus clearance therapy. Respir Care. 2007;52:1348-1358; discussion 1358-1361.

47. Nicolson CH, Stirling RG, Borg BM, Button BM, Wilson JW, and Holland AE: The long term effect of inhaled hypertonic saline $6 \%$ in non-cystic fibrosis bronchiectasis. Respir Med. 2012;106:661-667.

Received on December 18, 2017 in final form, April 1, 2018

Reviewed by: Adam Wanner Neil Alexis

Address correspondence to: Eva Polverino, $M D, P h D$ Servei de Pneumologia

Hospital Universitari Valld'Hebron Institut de Recerca Valld'Hebron Passeig de la Valld'Hebron, 119-129

Barcelona 08035

Spain

E-mail: eva.polverino@vhir.org 